\title{
Renal Involvement in Acute Gastroenteritis under 5 years of age in a Tertiary Care Hospital of Western Nepal - A prospective Observational Study
}

Badri Kumar Gupta ${ }^{1 *}$, Nand kishor Gupta ${ }^{2}$, Nikil Agrawal ${ }^{3}$, Binod Kumar Gupta ${ }^{1}$, Pradeep Chetri ${ }^{4}$

${ }^{1}$ Department of Pediatric, Universal College of Medical Sciences, Bhairahawa, Rupandehi, Nepal

${ }^{2}$ Department of Anatomy, Uttarpradesh Universal College of Medical Sciences, Saifai, Etawah, Uttar Pradesh, India

${ }^{3}$ Department of Paediatrics, Nepalese Army Institute of Health Sciences, Kathmandu, Nepal

${ }^{4}$ Department of Community Medicine, Universal College of Medical Sciences, Bhairahawa, Rupandehi, Nepal

*Corresponding author: Badri Kumar Gupta

\section{Abstract}

Acute renal failure is one of the important complications of acute gastroenteritis in children. Early intervention and proper fluid replacement may lessen this risk. Acute gastroenteritis (AGE) is an infection of the gastrointestinal tract that commonly affects young children. It causes an increase in loss of water and electrolytes due to vomiting and diarrhea resulting to dehydration, which is the main cause of mortality in children under five years with acute gastroenteritis. Therefore, this study were design to find out the incidence of AGE and the status of associated electrolyte derangements, renal involvementand other biochemical and haematological variables. Material and methods: This cross sectional study comprised of 100 cases of AGE in children aged <5 years of age. Demographic profile of the patients, details of diarrhea, and accompanying degree of dehydration (defined as per World health Organization criteria) with any associated complication were included. Various electrolyte parameters along with biochemical and hematological parameters (serum urea, serum creatinine, hemoglobin, and total leukocyte count) were investigated. Results: This study found that urea and creatinine were elevated in 29(29\%) and 62(62\%) of the cases respectively. Electrolyte disturbances were seen in $42 \%$ of cases among which hyponatremia was the most common electrolyte disturbance seen. Majority of patients had isonatremic dehydration 58\%. Hyponatremic dehydration was most common in some dehydration, which was $30 \%$. We found hypokalemia in $31 \%$ cases. Conclusion: Measurements of renal parameters early can help to predict the complications due to AGE and may help in the prevention of diarrhea related complications in children.

Keywords: Acute Kidney Injury; Child; Gastroenteritis. Electrolyte.

Copyright @ 2020: This is an open-access article distributed under the terms of the Creative Commons Attribution license which permits unrestricted use, distribution, and reproduction in any medium for non-commercial use (NonCommercial, or CC-BY-NC) provided the original author and source are credited

\section{INTRODUCTION}

Acute gastroenteritis is generally defined as a decrease in the consistency of stools (loose or liquid) and/or an increase in the frequency of evacuations (typically $\geq 3$ in 24 hours), with or without fever or vomiting; however, a change in stool consistency versus previous stool consistency is more indicative of diarrhea than stool number, particularly in the first months of life. Acute diarrhea typically lasts $<7$ days and not $>14$ days [1]. The term Gastroenteritis denotes infections of the gastrointestinal (GI) tract caused by bacterial, viral, or parasitic pathogens [2]. Worldwide, Gastrointestinal diseases are considered as common illnesses, the second most common cause for visits to physicians, and of morbidity and mortality among infants and children under five throughout the developing world. It has been estimated that in Latin America, Africa, and Asia depending on nutritional and socio - economic factors, a child's chance of dying by diarrheal illness before the age of five years can be as high as $50 \%$. In developing countries, a quarter of infants and childhood mortality is related to diarrhea. In Iraq, according to the annual report of the Iraqi Ministry of Health (M.O.H), the average of diarrheal patients visiting the medical and health care facilities related to M.O.H for the years 2009 and 2010 is 201 and 212 / 1000 child under five years of age. Also, it is estimated that the percentage of diarrhea cases to the total pathological cases requiring hospitalization in children less than five years of age is $19.1 \%$ for the year 2009 , and $24.9 \%$ for the year 2010[3]. Diarrheal disease occupied the second place among the top ten diseases in Nepal[4]. Poverty, illiteracy, lack of health care facilities at local level, demographical distribution and traditional beliefs are the major obstacles for getting proper and timely healthcare [5]. 
The main route of transmission of enteropathogens is faecal-oral through the ingestion of contaminated food or fluids or by direct person-to-person contact. The factors that increase the transmission of enteropathogens in developing countries include contaminated water and food, poor sanitation and hygiene, lack of breastfeeding, malnutrition, deficiencies in micronutrients like zinc or vitamin A, crowded environment, and living close to domestic animals. The key reservoirs of human enteropathogens are food, water and humans, but some of these infections (e.g. Salmonella, Campylobacter and Yersinia) are zoonoses that are transmitted from live animals, or by unsafe preparation of food. Children less than 2 years of age are particularly exposed to enteric pathogens, because of poor hygiene of hands and feet, and their explorative behaviour. When exposed, they often develop gastroenteritis due the lack of immunity induced by previous infections. The transition from breastfeeding, which provides some protection against enteropathogens, to formula or cow milk also increases the risk of gastroenteritis [6].

Other direct consequences of diarrhea in children include growth faltering, malnutrition, and impaired cognitive development in resource-limited countries [7]. Ramshackle housing, lack of piped water and sanitation, widespread fecal contamination of the environment, lack of personal hygiene, high incidence of malnutrition and infection, overcrowding of children under five years of age in one house have been found to be the contributing factors[8].

Childhood mortality rate is higher in families that are poor, living in rural areas and whose mothers lack basic education. Diarrhoea is about $13 \%$ higher in rural children than urban in the age group between 6 to 23 months. Moreover, there is a higher incidence of diarrhoea in children with uneducated mothers as compared to those whose mothers have some primary education. Children, especially infants, are more susceptible than adults to dehydration because of the greater basal fluid and electrolyte requirements per $\mathrm{kg}$ and as they are dependent on others to meet these demands.

Acute gastrointestinal illnesses are amongst the most common diseases worldwide: ranging from mild annoyances to devastating, dehydrating illnesses that can kill within hours [9]. AGE causes an increase loss of water and electrolytes through vomiting and diarrhea resulting to dehydration, which is the main cause of mortality in children [10]. Serum electrolyte and acid base disturbances are common in under-five children with acute diarrhea but may remain unrecognized, resulting in morbidity and sometimes mortality. Of the electrolyte abnormalities observed, hyponatremia and hypokalemia ranked first and second in frequency, respectively [11]. Timely recognition, a high index of suspicion and a thorough understanding of common electrolyte abnormalities is necessary to ensure their correction [12]. Episodes of acute diarrhoea occur most commonly in the first year of life, at a time when not only brain development is incomplete, but also when the wide variety of intestinal transport mechanisms which are concerned with the absorption and secretion of fluid and electrolytes are poorly developed[12]. Samadi et al. in their study in Bangladesh reported that $20.8 \%$ were hyponatremic, $72.8 \%$ isonatremic and $6.4 \%$ were hypernatremic[14].

During the past three decades, factors such as the widespread availability and use of Oral Rehydration Solution (ORS), improved rates of breastfeeding, improved nutrition, better sanitation and hygiene, and increased coverage of measles immunization are believed to have contributed to a decline in the mortality rate in developing countries. However, the morbidity from diarrheal diseases has remained relatively constant during the past two decades [7]. Urea showed good specificity, and anion gap was the most sensitive laboratory parameter for assessment of dehydration.

\section{MATERIAL AND METHODS}

This hospital based prospective observational cross sectional study were conducted in Universal College of Medical Sciences (UCMS), a tertiary care hospital in western Nepal over a period of six months (July-December 2019). Detailed history, examination and investigations wererecorded in specially designed proforma. Demographic profile of the patients, details of diarrhea such as duration, frequency, consistency of stools and accompanying degree of dehydration as per WHO criteria and any associated complication were recorded and investigated. Note were made about use of ORS before arriving to the hospital. A detailed examination were done by a pediatrician to assess the degree of dehydration, presence or absence of features suggestive of dyselectrolytemia and renal involvement.

The levels of serum sodium $\left(\mathrm{Na}^{+}\right)$, potassium $\left(\mathrm{K}^{+}\right)$, serum urea, serum creatinine, packed cell volume (PCV), hemoglobin $(\mathrm{Hb})$, with total leukocyte count (TLC) along with differential leukocyte count was investigated. Serum sodium and potassium were analyzed by biochemical analyzer (Ion Selective Method) based on the principle of electrochemistry. Similarly, serum urea and creatinine concentration were determined by kinetic urease-GLDH and alkaline picrate method respectively.Chest X-ray was done when required.

\section{Study definitions}

- Sodium level was considered normal between 135-145 mmol/L

- Potassium level was considered normal between 3.5- $5.5 \mathrm{mmol} / \mathrm{L}$

- Blood urea was considered normal in between 15-40 
$\mathrm{mg} / \mathrm{dl}$

- Serum creatinine was considerednormal in the range of $0.4-0.7 \mathrm{mg} / \mathrm{dL}$ in children (up to 5 years of age).

\section{Aims and objectives}

The aims and objectives of the present study were to study the incidence and outcome of renal involvement in acute gastroenteritis in children $<5$ years of age and to study the relation of severity of dehydration with renal injury.

\section{Inclusion Criteria}

- All children aged one month to 5 years acute gastroenteritis admitted in the pediatric department.

\section{Exclusion Criteria}

- Any child with history of diarrhea more than two weeks at the time of admission

- Children with dysentery (blood and mucus in stool)

- Children with known pre-existing renal disease, children who underwent renal replacement therapy before admission.

\section{STATISTICAL ANALYSIS}

The data were enter in predesign proforma and was analyzed with SPSS version 22 (Statistical Package for the Social Science) software. Frequency of the qualitative variables were presented in the percentage. Value of continuous variables were present, as mean \pm SD. Data were analyzed using Chi square test for qualitative variables and analysis of variance (ANNOVA) were use for comparison of mean of continuous variables. Odd's ratio were calculated for risk assessment. Status of hydration biochemical variables were analyze by Spearman's rho correlation coefficient. $\mathrm{P}$ value of less than 0.05 was considered statistically significant.

\section{RESULTS}

Out of total 175 admitted gastroenteritis cases, 100 children were enrolled in the study as per the inclusion criteria. Out of 100 cases enrolled 66 (66\%) were males and $34(34 \%)$ were females with male to female ratio of 1.94:1.

Table; 1

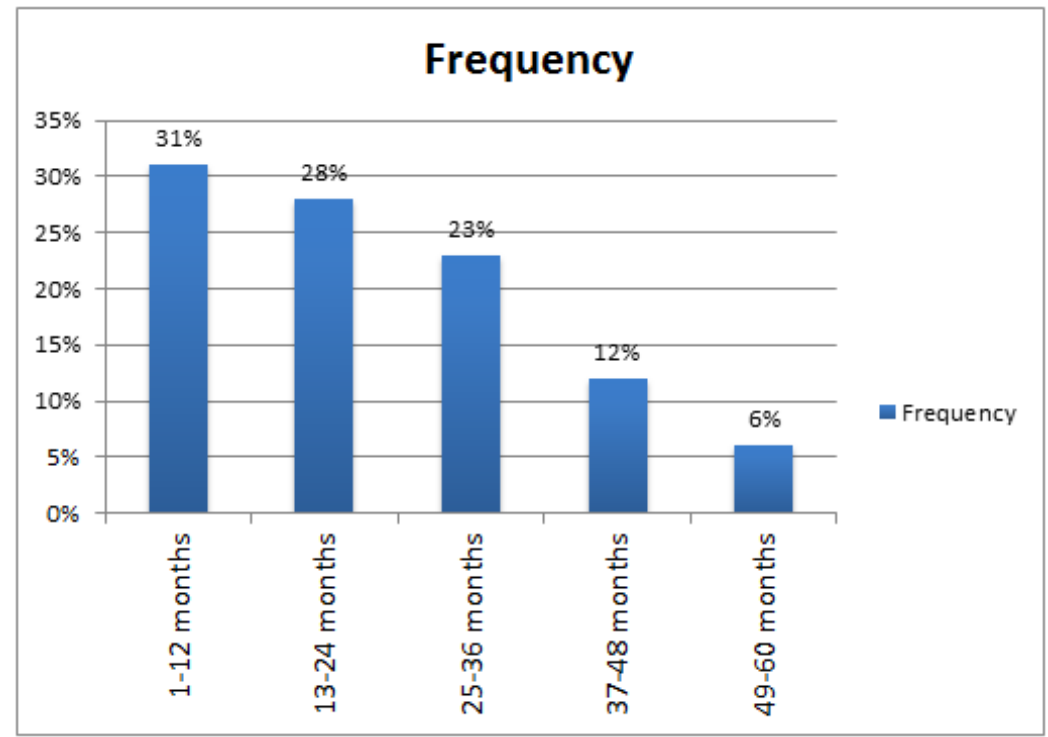

Fig-1: Frequency of Patients Based on Different Age Group

Figure 1 shows that children aged 1-12 months had the highest incidence of diarrhea $(31 \%)$, which were followed by 13-24 months (28\%), 25-36 months (23\%), and 37-48 months (12\%). The age group 49-60 months had the lowest incidence (6\%).

Table-1: Level of Biochemical and Hematological Parameters in Different Age Groups (Months)

\begin{tabular}{|l|l|l|l|l|l|l|}
\hline & $\mathbf{1 - 1 2}$ & $\mathbf{1 3 - 2 4}$ & $\mathbf{2 5 - 3 6}$ & $\mathbf{3 7 - 4 8}$ & $\mathbf{4 9 - 6 0}$ & P-value \\
\hline $\mathrm{Na}^{+}$ & $136.34 \pm 4.66$ & $136.47 \pm 3.82$ & $134.16 \pm 4.40$ & $137.15 \pm 2.92$ & $135.70 \pm 3.93$ & 0.20 \\
\hline $\mathrm{K}^{+}$ & $4.00 \pm 0.76$ & $3.92 \pm 0.60$ & $3.63 \pm 0.55$ & $4.00 \pm 0.52$ & $3.71 \pm 0.59$ & 0.23 \\
\hline Urea & $35.58 \pm 25.51$ & $32.57 \pm 22.58$ & $44.34 \pm 26.8$ & $30.66 \pm 17.47$ & $40.50 \pm 23.40$ & 0.39 \\
\hline Creatinine & $0.82 \pm 0.30$ & $0.87 \pm 0.41$ & $1.10 \pm 0.51$ & $0.83 \pm 0.13$ & $1.01 \pm 0.41$ & 0.08 \\
\hline $\mathrm{Hb}$ & $10.08 \pm 1.52$ & $10.57 \pm 1.48$ & $10.82 \pm 1.92$ & $11.86 \pm 1.47$ & $10.50 \pm 1.57$ & 0.03 \\
\hline TLC & $12006.45 \pm 6261.51$ & $13025.00 \pm 6928.07$ & $14267.39 \pm 6363.70$ & $12291 \pm 4592.17$ & $14600.00 \pm 6127.96$ & 0.69 \\
\hline PCV & $31.16 \pm 4.21$ & $33.85 \pm 6.61$ & $32.72 \pm 5.61$ & $35.96 \pm 3.77$ & $31.75 \pm 4.50$ & 0.08 \\
\hline
\end{tabular}




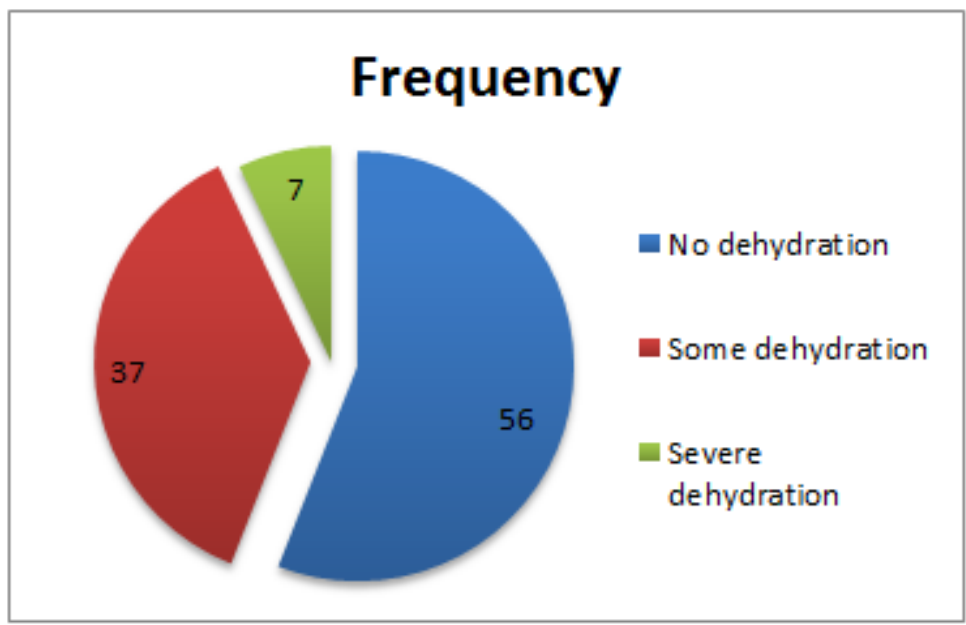

Fig-2: Degree of Hydration Based on WHO Criteria

Figure 2 shows that out of 100 cases enrolled, no dehydration was observed in highest number of cases
(56\%) followed by some dehydration (37\%) and severe dehydration $(7 \%)$.

Table-2: Frequency of Patients in Different Status of Electrolytes

\begin{tabular}{|c|c|c|}
\hline Biochemical parameters & & Frequency \\
\hline Serum Sodium & Normal & $58 \%$ \\
\cline { 2 - 3 } & Low & $42 \%$ \\
\hline \multirow{3}{*}{ Serum Potassium } & Normal & $67 \%$ \\
\cline { 2 - 3 } & Low & $31 \%$ \\
\cline { 2 - 3 } & High & $2 \%$ \\
\hline \multirow{2}{*}{ Serum Urea } & Normal & $71 \%$ \\
\cline { 2 - 3 } & High & $29 \%$ \\
\hline Serum Creatinine & Normal & $38 \%$ \\
\cline { 2 - 3 } & High & $62 \%$ \\
\hline
\end{tabular}

Table 2 depicts the frequency of different biochemical parameters in children with AGE. Majority of admitted children with AGE had normal urea (71\%) and raised creatinine $(62 \%)$. Urea were only raised in $29 \%$ of cases.

Table-3: Comparison of renal parameters with hydration status

\begin{tabular}{|l|l|l|l|l|}
\hline Renal parameters & Group 1 & Group 2 & Group 3 & $p$ value \\
\hline Urea $(\mathrm{mg} / \mathrm{dl})$ & $25.69 \pm 12.59$ & $43.05 \pm 23.98$ & $80.26 \pm 30.56$ & 0.001 \\
\hline Creatinine $(\mathrm{mg} / \mathrm{dl})$ & $0.75 \pm 0.14$ & $0.97 \pm 0.38$ & $1.81 \pm 0.56$ & 0.001 \\
\hline
\end{tabular}

Group 1= No dehydration, Group 2= Some dehydration, Group 3= Severe dehydration

Table 3 shows serum urea and creatinine were significantly high in severe dehydration $[80.26 \pm 30.56$ $\& 1.81 \pm 0.56]$ as compared to some dehydration
$[43.05 \pm 23.98 \& \& \quad 0.97 \pm 0.38]$ and no dehydration $[25.69 \pm 12.59 \& 0.75 \pm 0.14]$.

Table-4: Odd's Ratio of renal parameters and oral rehydrating solution use

\begin{tabular}{|l|l|l|}
\hline Renal parameters & \multicolumn{1}{|c|}{ OR $(\mathbf{C I})$} & p-value \\
\hline Urea & $0.399(0.130-1.2290)$ & 0.095 \\
\hline Creat & $0.357(0.094-1.360)$ & 0.100 \\
\hline
\end{tabular}

Table 4 shows the odd's ratio of renal parameters seen in the present study in relation to ORS use. The study suggested that there were no significant changes in renal parameters with adequate ORS use.

\section{DISCUSSION}

Studies in developing countries have shown that children in the first 2 years of life may have up to 10 episodes of diarrheal disease, often with significant mortality. The total incidence of acute gastroenteritis were $7.8 \%$ in the present study. 
Poo et al.[15] reported that acute gastroenteritis was responsible for $6 \%$ of the total pediatric admission, which was comparable to this study(178 diarrheal cases out of 2236 pediatric admission).Ramanaiah et al. [16] found that the incidence of acute diarrheal disease among under five children admitted as $12.1 \%$, which was higher than the present study. In another study done by Yilgwan et al. [17] in Nigeria, the incidence of acute gastroenteritis was only $2.7 \%$, which was very much lower than the present study.

The differences in the incidence between the present study and other studies may be attributed to the differences in the time, setting of the study and region.

A study done by Stanly et al. [18] reported that the average number of times of diarrhea per child per day was 5 with a minimum number of 3 and maximum of 10 . Kolahi et al. [19] reported the mean frequency of diarrhea was $5.1 \pm 5$ episode per day.

In the present study, majority of the admitted cases $73,(73 \%)$ suffered from 1-2 days of diarrhea before admission followed by $25(25 \%)$ from 3-4 days. Only 2 (2\%) cases suffered from diarrhea from 5-6 days. Similar result was seen in a study done by Poo et al. [15] who reported $50 \%$ had duration of symptoms of two days or less before admission, while $76 \%$ had symptoms for less than three days.

A study done by Poo et al. [15] reported that $37 \%$ had vomiting less than 5 times per day whereas $29 \%$ had vomiting 5-10 times per day. In the same study, it was reported that $24 \%$ had vomiting more than 10 times per day.

In this study, the most common chief complaint was vomiting (88\%), followed by fever (74\%), distention of abdomen (26\%), thirst (23\%), scanty urination $(23 \%)$ and convulsion $(2 \%)$. The sensorium was affected in $42 \%$ of the cases; $35 \%$ irritable and $7 \%$ lethargic.

In a study done by Azemi et al. [20] in Kosovo, loose stool was the most frequent symptom seen in $98.59 \%$ cases, followed by vomiting in $88.02 \%$ cases, which was similar to our study, and fever in $54.22 \%$ cases. Poo et al. in their study reported that vomiting was present in more than $90 \%$ of children on admission and loose stool was present in all children on admission, which was comparable to our study. Similarly, Khalili et al. [21] reported that vomiting and fever were the most frequent concomitant symptoms during presentation in hospitalized children.

The present study shows that no dehydration was observed in most number of cases $(n=56,56 \%)$, followed by some dehydration $37(37 \%)$ and severe dehydration $7(7 \%)$. Tavakolizadeh et al. [22] in their study reported that among children admitted with acute gastroenteritis, $64.9 \%$ had no dehydration, $32.7 \%$ had some dehydration, which was comparable to our study, and only $2.4 \%$ cases had severe dehydration. A study done by Azemi et al. [20] in Kosovo reported some dehydration to be present in $42.29 \%$ of cases which was comparable to our study and severe dehydration in $14.08 \%$ cases.

These findings are in agreement with prior studies demonstrating that no dehydration is present in majority of hospitalized children with acute diarrhea. The main reason for some dehydration being seen in majority of the cases following no dehydration in our study may be because of the lack of knowledge among most of the mother's about the use of ORS in diarrhea. As it has been noted that only $15 \%$ of the cases received ORS prior to hospitalization.

In our study majority of the patients had intact sensorium in mild and when dehydration was not present whereas in severe form of dehydration majority of the patients had altered sensorium. It is seen that when there is deterioration in the status of hydration in the children, there is worsening of the sensorium as well.

In the present study, electrolyte disturbances were seen in $42(42 \%)$ cases while $58(58 \%)$ cases had no electrolyte disturbance. The most common electrolyte disturbance was hyponatremia $(42 \%)$ followed by hypokalemia in $31 \%$. Mixed electrolytes disturbances were seen in $33(33 \%)$ of the cases out of which the most common was hyponatremia with hypokalemia seen in $75.75 \%$ cases followed by normal sodium with hypokalemia $(6 \%)$. Normal sodium with hyperkalemiawas seen in $2 \%$ cases. In this study isolated hyponatremia was seen in $17 \%$ cases with isolated hypokalemia in $6 \%$ and isolated hyperkalemia was seen in $2 \%$ cases. Shah et al. [23] in their study done in pediatric ward of BPKIHS, Dharan reported $79 \%$ of children suffering from diarrhea to have some form of electrolyte abnormalities, which was found to be higher than our study. In the same study, the second common electrolyte disturbance was hypokalemia (46\%), which was isolated in $14 \%$ and associated with hyponatremia in 26\%. Similarly, Ukarapol et al. [24] in Thailand found electrolyte abnormalities in $69.8 \%$ of children having acute diarrhea.

Out of 100 cases enrolled in the study, 71(71\%) had normal urea, whereas $29(29 \%)$ had increased serum urea level. The present study shows that $62(62 \%)$ had increased serum creatinine, while 38 (38\%) had normal serum creatinine level. Further, the increase in serum urea was seen in more number of cases that is in $20 \%$ in some dehydration followed by severe dehydration $(6 \%)$ whereas only $3 \%$ of the cases had increase urea in no dehydration. Moreover, serum creatinine was found to 
be increased in no, some and severe dehydration that is, in $27 \%, 28 \%$ and $7 \%$ respectively.

Malaki et al. [25] found that BUN level was significantly higher in severe dehydration compared to mild or moderate dehydration whereas there was no difference in the serum creatinine level between mild and moderate dehydration but in severe dehydration creatinine level was significantly higher. Similar result was seen in the study conducted by Ukarapol et al. [24] where $24 \%$ of the patient had increase BUN and the BUN correlated statistically with the degrees of dehydration. On the contrary, Tavakolizadeh et al. [22] in their study using children with acute gastroenteritis reported the most common biochemical abnormality to be abnormal serum creatinine, which was reported in $14.2 \%$ cases. Gauchan et al. [26] reported that out of 188 cases high blood urea was found in 20 of severe dehydration as compared to 63 of some and 15 of no dehydration. Further high serum creatinine was found in 18 of severe, 79 of some and 17 of no dehydration $[8,6]$.

Thus, serum urea, creatinine and BUN should be done in all children having acute gastroenteritis with no, some and severe dehydration as it may be difficult to predict which children have significant abnormalities. But these laboratory tests should not be considered as definitive predictors of dehydration.

In the present study, only $15 \%$ cases had received ORS prior to hospitalization while $85 \%$ had not. It was observed that out of 15 cases that had been on ORS, hyponatremia was seen in 10 cases; as compared to 32 cases who had not been on ORS. Likewise, 10 cases had hypokalemia who had received ORS as compared to 21 cases who had not received ORS. Similarly, 22 patients had high serum urea level and 50 patients had high creatinine level when ORS was not used.

Gauchan et al. [26] reported that out of 72 children who had been on ORS prior to admission, hypernatremia was seen in $14(19.4 \%)$ and hyponatremia in $6(8.3 \%)$; as compared to $13.8 \%$ and $12.9 \%$ respectively in those who had not been on ORS.

Seddique et al. [27] reported that the use of ORS, by $65 \%$ of the subjects in their study. Similarly, in a study done by Ukarapol et al. about $60.4 \%$ had a history of ORT before admission, which was higher compared to this study. Rasania et al. [28] reported that $69.8 \%$ of mothers knew the role of ORS during diarrhea whereas $46 \%$ of them never used ORS. In the same study it was reported that only one-third $(38.7 \%)$ of the subjects could tell the correct method of ORS. Mohsin et al. [29] reported that $76 \%$ mothers had correctly prepared ORS and 24\% had prepared incorrectly.

ORS has been considered the "gold standard" of oral rehydration therapy. However, a Cochrane review which included seventeen trials (1811 participants) did not show a significant advantage of ORS over intravenous hydration in the outcome in dehydration associated with diarrhea in children[8,7]. According to Nepal Demographic Health Survey (NDHS) 2011, the percentage of children treated with ORS has increased from $29 \%$ in 2006 to $39 \%$ in 2011 [18].

ORT is the cheap, simple and effective way to prevent/treat dehydration caused by diarrhea. Timely and appropriate use of ORS is associated with reduced need for hospitalizations, intravenous infusions, risk of dehydration and electrolyte abnormalities. Improper preparation and intake of ORS hampers the prevention and treatment of diarrheal diseases, and may further lead to electrolyte disturbances and contribute to morbidity and mortality. Thus, lack of use of ORS hampers the prevention and treatment of diarrheal diseases, and may further lead to electrolyte disturbances and contribute to morbidity and mortality. Thus, lack of use of ORS in cases of diarrhea is considered as a risk factor as identified by the present study.

\section{CONCLUSION}

The level of serum sodium and potassium decreased and urea and creatinine increased with severity of dehydration. In this study majority of cases $(85 \%)$ were not on ORS and had low sodium and potassium. Thus, electrolyte disturbances are one of the major concerns among children with acute gastroenteritis and timely recognition and correction is necessary to prevent morbidity and mortality. Early diagnosis and urgent management of gastroenteritis and dehydration can prevent AKI.

\section{REFRENCES}

1. Guarino A, Ashkenazi S, Gendrel D, Lo Vecchio A, Shamir R, Szajewska H. European Society for Pediatric Gastroenterology, Hepatology, and Nutrition/European Society for Pediatric Infectious Diseases Evidence-Based Guidelines for the Management of Acute Gastroenteritis in Children in Europe: Update 2014. JPediatr GastroenterolNutr. 2014 Jul;59(1):132-52.

2. Bhutta ZA. Acute Gastroenteritis in Children. Kliegman RM, Behrman RE, Stanton BF, Schor NF, Geme JWS, editors. Nelson Textbook OfPaediatrics. Philadelphia: Saunders Elsevier; 19th Edition; 2011:1323.

3. Ibrahim B.M. Isolation of Some Microbial Agents That Cause Acute Gastroenteritis in Children. J Fac Med Baghdad. 2012;54(2):218-222.

4. Pokharel M, Sherchand JB, Upreti HC, Katuwal A, Gauchan P. A Perspective Study on the Etiology of Diarrhea in Children Less than 12 Years of age attending Kanti Children's Hospital J. Nepal Paediatr. 2009 jan; 29(1):10-16.

5. Ansari M, Palaian S, Izham M, Ibrahim M. The Millennium Development Goals Report: United 
Nations Department of Economic and Social Affairs (DESA) 2008.The Role of Mothers in the Management of Childhood Diarrhoea in Nepal. Australasian Medical Journal.2009; 1(14):235.

6. Kabayiza JC, Acute Gastroenteritis in Rwandan Children under five years of age Investigated by Real-time PCR, Depart. ofInfect. Disease, Gothenburg University. 2014.P-4.

7. Farthing M,Salam M, Lindberg G.Acute Diarrhea in adults and children: A Global Perspective. World Gastroenterology organisation, Feb.2012.

8. Karki A, Tiwari BR. Prevalence of Acute diarrhea in Kathmandu Valley. J Nepal Med Assoc. 2007;46(168):177.

9. Ukey UU, Shekhar R, Ganesh L.Seasonal Patterns in Acute Gastroenteritis in a Hospital at Maharashtra, India. IJHSR. 2012; 1(2):101-108.

10. Wambui NM. Barriers to Appropriate Home Management of Acute Gastroenteritis by Caregivers of Children under Five Years Attending Mbagathi District Hospital.2014 nov. P-5.

11. Onyiriuka A N, Iheagwara E C. Serum Electrolyte Profiles of under-five Nigerian Children admitted for Severe Dehydration due to Acute Diarrhea. Niger J Health Sci. 2015;15:14-7.

12. Shah GS, Das BK, Kumar S, Singh MK, Bhandari GP. Acid Base and Electrolyte Disturbance in Diarrhea. KUMJ.2007 Jan-Mar;5(17):60-62.

13. Whyte LA and Jenkins HR. Pathophysiology of diarrhea(Review).Paediatrics and Child Health.2012;22(10):443-447.

14. Samadi AR, Wahed MA, Islam MR, Ahmed SM. Consequences OfHyponatraemia and Hypernatraemia in Children with Acute Diarrhoea in Bangladesh. British Medical Journal (Clinical research ed). 1983;286(6366):671-673.

15. Poo MI, Lee WS. Admission to hospital with childhood acute gastroenteritis in Kuala Lumpur, Malaysia. Med. J. Malaysia 2007 Aug; 62(3):189-93.

16. Ramanaiah VD, Arumugam SN A, Prabhu R. Sociodemographic profile of acute diarrhoeal diseases in a tertiary care hospital, Tirupati. IOSR Journal of Dental and Medical Sciences (IOSR-JDMS). 2015 May;14(5):29-32.

17. Yilgwan CS, Okolo SN. Prevalnce of diarrhea disease and risk factors in Jos University Teaching Hospital, Nigeria. Ann Afr Med. 2012;11(4):217-21.

18. Stanly AM, Sathiyasekaran BWC, Palani G. A Population based study of Acutediarrhoea among children under 5 years in a rural community in South India. Sri Ramachandra Journal of Medicine. 2009 Jan - June;1(1):1-6.

19. Kolahi AA, Nabavi M, Sohrabi MR. Epidemiology of acute diarrheal diseases among children under 5 years of age in Tehran, Iran. Iranian Journal of Clinical Infectious Diseases. 2008;3(4):193-98.

20. Azemi M, Berisha M, Ismaili-Jaha V, Kolgeci S. Socio-demographic, Clinical and Laboratory features of Rotavirus Gastroenteritis in Children Treated in Pediatric Clinic. Mat Soc Med. 2013 Mar; 25(1): 9-13.

21. Khalili B, Shahabi G, Khalili M, Mardani M, Cuevas LE. Risk factors for hospitalization of children with diarrhea in Shahrekord, Iran. Iranian Journal of Clinical Infectious Diseases. 2006; 1(3):131-6.

22. Tavakolizadeh $R$, Sadeghi $M$, Namiranian $N$, Fahimi D, Barkhordari M. Blood chemical analysis in children with acute gastroenteritis, When Is It Useful? J Ped nephrology 2013; 1 (2):65-9.

23. Shah GS, Das BK, Kumar S, Singh MK, Bhandari GP. Acid Base and Electrolyte Disturbance in Diarrhea. KUMJ.2007 Jan-Mar;5(17):60-62.

24. Ukarapol N, Wongsawasdi L, Chartapisak W, Opastirakul S. Electrolyte Abnormalities In Children With Acute Diarrhea. Chiang Mai Med Bull. 2002; 41(1):7-12.

25. Malaki Majid. Clinical versus laboratory for estimating of dehydration severity. Annals of Tropical Medicine and Public health year. 2012; 5(6):559-62.

26. Gauchan E, Malla KK. Relationship of Renal Function Tests and electrolyte levels with severity of dehydration in acute diarrhea. J Nepal Health Res Counc. 2015 Jan - Apr;13(29): 84-9.

27. Ranjbar SA, Bavafa B. Iranian mother's child feeding practices during diarrhea: A study in Kerman. Pak J. Nutrion.2007; 6(3):217-19.

28. Rasania SK, Singh D, Pathi S, Matta S \& Singh S. Knowledge and Attitude of mothers about Oral Rehydration Solution in few urban slum of Delhi. Health and Population - Perspectives and Issues.2005; 28(2): 100-107.

29. Mohsin A, Raza AB, Ahmad TM. Knowledge, Attitude and Practices of the mothers regarding Oral Rehydration Solution, Feeding and use of drugs in childhood diarrhoea. P J M H S.2012Jan-Mar. 6(1):108. 